This is a self-archived version of an original article. This version may differ from the original in pagination and typographic details.

Author(s): Chakraborty, Subrata; Heikkilä, Tero

Title: Thermoelectric radiation detector based on a superconductor-ferromagnet junction : Calorimetric regime

Year: 2018

Version: Published version

Copyright: ㄷ AIP Publishing, 2018

Rights: In Copyright

Rights url: http://rightsstatements.org/page//nC/1.0/?language=en

Please cite the original version:

Chakraborty, S., \& Heikkilä, T. (2018). Thermoelectric radiation detector based on a superconductor-ferromagnet junction : Calorimetric regime. Journal of Applied Physics, 124(12), Article 123902. https://doi.org/10.1063/1.5037405 
Thermoelectric radiation detector based on a superconductor-ferromagnet junction: Calorimetric regime

Subrata Chakraborty, and Tero T. Heikkilä

Citation: Journal of Applied Physics 124, 123902 (2018); doi: 10.1063/1.5037405

View online: https://doi.org/10.1063/1.5037405

View Table of Contents: http://aip.scitation.org/toc/jap/124/12

Published by the American Institute of Physics

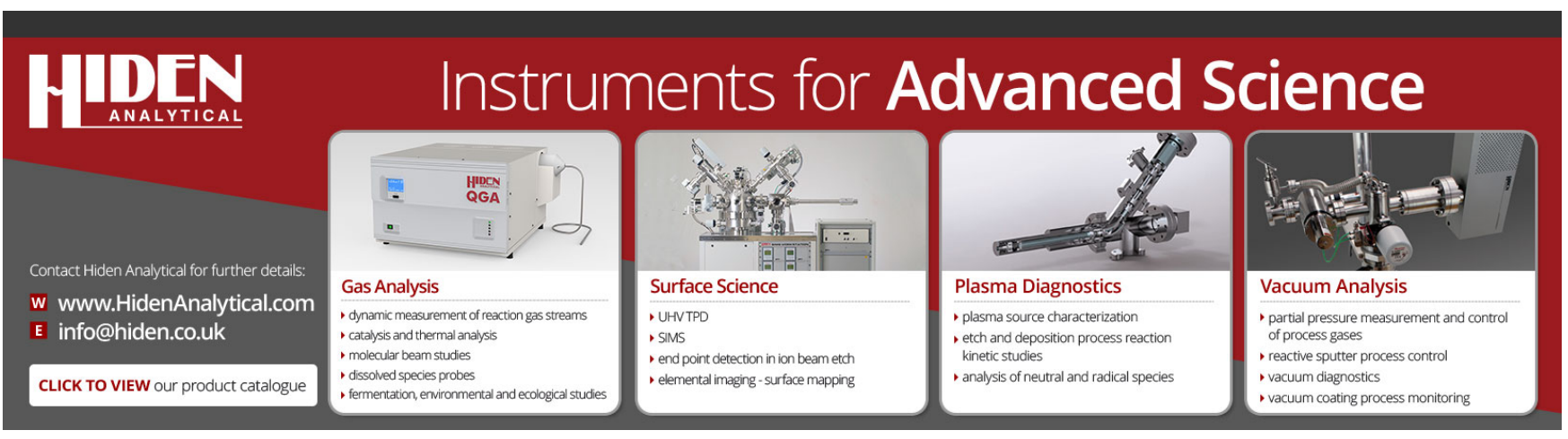




\title{
Thermoelectric radiation detector based on a superconductor-ferromagnet junction: Calorimetric regime
}

\author{
Subrata Chakraborty ${ }^{\mathrm{a})}$ and Tero T. Heikkilä \\ Department of Physics and Nanoscience Center, University of Jyväskylä, P.O. Box 35 (YFL), FI-40014 \\ Jyväskylä, Finland
}

(Received 24 April 2018; accepted 2 September 2018; published online 25 September 2018)

\begin{abstract}
We study the use of a thermoelectric junction as a thermal radiation detector in the calorimetric regime, where single radiation bursts can be separated in time domain. We focus especially on the case of a large thermoelectric figure of merit $Z T$ affecting significantly, for example, the relevant thermal time scales. This work is motivated by the use of hybrid superconductor/ferromagnet systems in creating an unprecedentedly high low-temperature ZT even exceeding unity. Besides constructing a very general noise model which takes into account cross correlations between charge and heat noise, we show how the detector signal can be efficiently multiplexed by the use of resonant LC circuits giving a fingerprint to each pixel. We show that for realistic detectors operating at temperatures around 100 to $200 \mathrm{mK}$, the energy resolution can be as low as $1 \mathrm{meV}$. This allows for a broadband single-photon resolution at photon frequencies of the order of or below $1 \mathrm{THz}$. Published by AIP Publishing. https://doi.org/10.1063/1.5037405
\end{abstract}

\section{INTRODUCTION}

Some of the most sensitive sensors of electromagnetic radiation are based on using superconducting films absorbing the radiation and measurement systems converting this process into detectable electronic signal. The best-studied example of such sensors is the superconducting transition edge sensor (TES), ${ }^{1}$ which has already been used for many types of applications, such as in security imaging, ${ }^{2}$ materials analysis, ${ }^{3,4}$ and cosmic microwave background radiation detection. ${ }^{5,6}$ In TES sensors, the absorbed radiation heats the electrons above the critical temperature $T_{c}$ of superconducting films and results in measurable changes in the film resistance. This resistance is often read out by utilizing an applied bias voltage or current, ${ }^{7}$ fixing the operating point close to $T_{c}$, and allowing for additional read-out features such as electrothermal feedback and bias-based multiplexing strategies. ${ }^{3}$ However, the presence of bias-induced dissipation also leads to an overall heating of the system and increases the thermal noise, thus reducing the sensitivity. In addition, in multi-pixel systems, fabricating bias lines for each pixel becomes a technological challenge. Another important sensor in this context is the kinetic inductance detector (KID $)^{8-12}$ based on the read-out of the kinetic inductance signal in superconducting microwave resonators. Also, KIDs require probe signals for read-out, resulting in increased dissipation within the pixels. As the desire in many applications is to further increase the number of detector pixels, ${ }^{13}$ such probe-based sensors become increasingly difficult to operate.

In general, one would prefer to only have the effect of the coupling between radiation and the detector in the

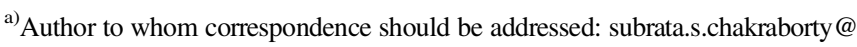
jyu.fi
}

measured signal and therefore to get rid of the probe signal. This desire can be achieved with a thermoelectric detector (TED) ${ }^{14-18}$ where the absorption of radiation leads to a temperature difference, which creates a measurable thermoelectric current or voltage. In this work, we consider such thermoelectric detectors. They have indeed the advantage of the lack of probe signals. However, for most systems, the thermoelectric effects are very weak, and therefore sensitivities close to those of TES and KID cannot be expected. This changed with the discovery of the giant thermoelectric effect in superconductor-ferromagnet hybrids, ${ }^{19-21}$ which can in principle be utilized to create sub-Kelvin thermoelectric heat engines with figures of merit ZT exceeding even those of the best thermoelectric devices. Hence, such systems may offer sensitivities rivaling those of TES and KID sensors, but without the need of probe signals. ${ }^{18}$

Compared to probe-based sensors, there are also issues with the proper read-out of the signal, as many existing multiplexing strategies are based on modulating the probe signal. We address this here by analyzing in detail the use of the thermoelectric detector in the calorimetric regime, where radiation arrives at bursts separated by long times compared to the relevant time scales of the detector. This is opposite to the bolometric regime analyzed in earlier work. ${ }^{18}$ In contrast to many recent works ${ }^{15,16}$ utilizing an ad hoc noise model, we derive the energy resolution of such a thermoelectric calorimeter by taking into account all the relevant noise terms, including the cross correlation of heat and current noises in the thermoelectric junction, as required by the linear response theory. As a result, we obtain the energy resolution and the relevant thermal time scales of the calorimeter modified by the large ZT, especially at low temperature, where the heat conduction of the detector is mostly governed by the electronic degrees of freedom. We also analyze the resulting time-dependent thermoelectrically generated current profile in various parameter regimes. 


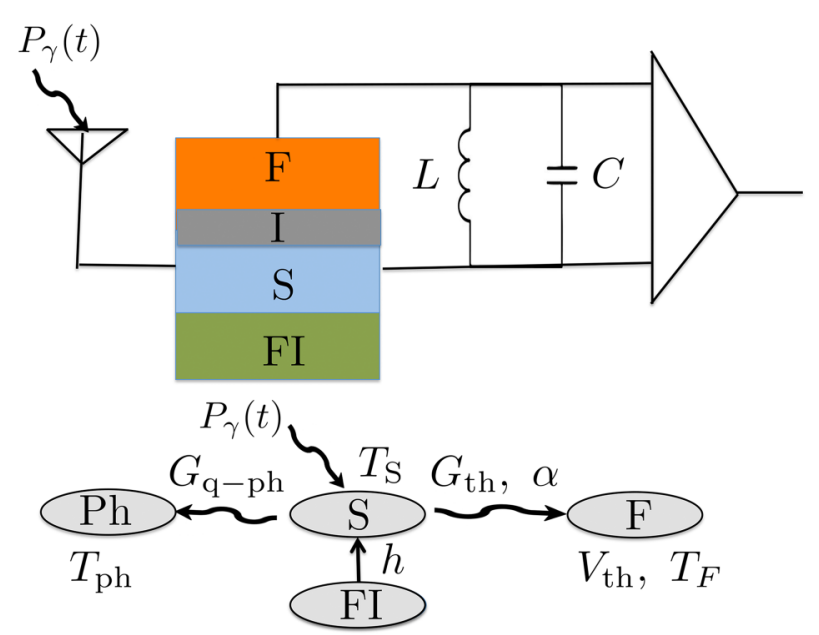

FIG. 1. Schematic of the thermoelectric detector based on superconductor (S) and ferromagnetic (F) electrode a spin-filter junction. $\mathrm{S}$ is also coupled with ferromagnetic insulator (FI) which provides a spin splitting exchange field to S. I is an insulating layer and $P_{\gamma}(t)$ is the time dependent power of incident radiation which needs to be detected. $T_{S}, T_{F}$, and $T_{\mathrm{ph}}$ are the electronic temperature of the superconducting film, the electronic temperature of the ferromagnetic electrode, and phonon temperature of the superconducting film, respectively.

In this work, we consider a single pixel of the thermoelectric detector based on a superconducting film and the ferromagnetic junction, ${ }^{18}$ as depicted in Fig. 1. The single pixel of the proposed detector is built with an element made of a thin film of a superconductor-ferromagnetic insulator (S-FI) bilayer coupled to superconducting antennas via a clean (Andreev) contact. Next, the S-FI bilayer is further coupled with a nonsuperconducting electrode via a spin filter such as a ferromagnetic metal $(F)$. The current injected into the ferromagnetic electrode or the voltage between the superconductorferromagnet (SF) tunnel junction due to the incident radiation power can be detected by a SQUID current amplifier or a field effect transistor such as HEMT, respectively. The ferromagnetic insulator (FI) which is in contact with the superconductor exerts a spin-splitting exchange field, $h$, into the superconductor. Below a certain critical value, this exchange field does not have a large effect on the superconducting gap, $\Delta,{ }^{22,23}$ but it has a major effect on the spin-dependent density of states. Another alternative hybrid structure of the detector in this context can be thought of as superconductor-ferromagnetic insulator-normal metal $(\mathrm{S} / \mathrm{FI} / \mathrm{N})$. In that case, the FI plays a dual role of providing both the spin-splitting field via the magnetic proximity effect and the spin-polarized tunneling required for the thermoelectric effect. ${ }^{21}$ However, the tunneling resistance in such junctions is often larger than in native oxide junctions, leading to somewhat smaller thermoelectric currents. $^{24,25}$ To prevent heat leaking to the antenna, it must be fabricated from a material with the superconducting gap $\Delta_{A}$ far exceeding the gap $\Delta$ of the absorber film.

In what follows, we first study the generic thermoelectric detectors in the calorimetric regime within the linear response regime. Then we analyze the energy resolution of the detector in the frequency domain with the idea of optimal filtering. After this, we concentrate on the superconductor-ferromagnet detector and study the energy resolution vs. bath temperature and the exchange field induced into the superconductor. Finally, we estimate the practically feasible energy resolution in such detectors.

\section{THEORY}

Here, we study the thermoelectric detector in the calorimetric regime, where the process of relaxation of the detector is much faster than the arrival of the consecutive incident pulses of energies to the detector. In what follows, we first consider the heat balance equation of a generalized thermoelectric detector within linear response assumption given by ${ }^{18}$

$$
C_{h} \frac{d \Delta T(t)}{d t}=P_{\gamma}(t)-G_{\mathrm{th}}^{\mathrm{tot}} \Delta T(t)-\alpha V_{\mathrm{th}}(t)
$$

where $P_{\gamma}(t)$ is the power of the incident radiation, $V_{\mathrm{th}}(t)$ is the time dependent voltage between the ferromagnetic electrode and the superconductor, $C_{h}$ is the heat capacity of the absorber, and $\alpha$ is the response coefficient for the Peltier heat current. In Eq. (1), $G_{\text {th }}^{\text {tot }}=G_{\mathrm{q}-\mathrm{ph}}+G_{\mathrm{th}}$ represents the total heat conductance of the superconducting film to the heat bath, and $G_{\mathrm{q}-\mathrm{ph}}$ and $G_{\text {th }}$ stand for the heat conductance of the quasiparticles in the superconductor to the phonons and to the ferromagnetic electrode, respectively. Another possible (spurious) heat conduction channel could be due to quasiparticle-magnon scattering, but we disregard it below as it depends on the microscopic details of the magnets. The quantity $\Delta T(t)=T_{S}(t)-T$ is the change of electronic temperature in the superconductor due to the incident power, where $T_{S}(t)$ is the time dependent temperature of the superconductor and $T$ is the bath temperature. Often in room-temperature thermoelectric devices, the bottleneck of heat transport lies in the heat conductance of the phonons to their bath. At low temperatures, however, this heat conductance is typically much larger than that related to electron-phonon coupling. ${ }^{7}$ As a result, we may assume $T_{\mathrm{ph}}=T_{F}=T$ so that phonons already reside at the bath temperature. The mechanism of the heat conduction is thus dominated by the heat flow by the electronic degrees of freedom of the superconductor with the electronic degrees of freedom of the ferromagnetic electrode and the phonons of the superconductor at low temperature as represented at bottom portion of the Fig. 1 by wiggly lines. Equation (1) in the frequency $(\omega)$ space gives the following solution for the change of temperature, $\Delta T(\omega)=\left[P_{\gamma}(\omega)-\alpha V_{\text {th }}(\omega)\right] /\left(G_{\text {th }}^{\text {tot }}+i \omega C_{h}\right){ }^{26}$ Next, within linear response assumption, we consider the thermoelectric current from the superconductor to the ferromagnetic electrode, ${ }^{18}$

$$
I_{\mathrm{th}}(t)=-\frac{\alpha}{T} \Delta T(t)-G V_{\mathrm{th}}(t)
$$

where $G$ is the conductance of the thermoelectric junction. This thermoelectric current through the thermoelectric junction ultimately reaches an amplifier. Disregarding the back-action noise from the amplifier and considering the amplifier as a capacitor or an inductor, the thermal current through the amplifier due to the thermoelectric voltage, $V_{\text {th }}$, in frequency $(\omega)$ space is $I_{\text {th }}(\omega)=V_{\text {th }}(\omega)[i \omega C+1 /(i \omega L)]$, where $C$ and $L$ are the 
capacitance and inductance of the detection circuit, respectively. Here $L$ and $C$ can represent elements that have been designed on purpose for identifying the pixel (see below). Next, using Eqs. (1) and (2), along with the expression of the current through the amplifier, we obtain the voltage across the thermoelectric junction $V_{\mathrm{th}}(\omega)=\lambda_{V}(\omega) P_{\gamma}(\omega)$, where $\lambda_{V}(\omega)=\alpha$ / $\left[\alpha^{2}-Y_{\text {th }}^{\text {tot }}(\omega) Y_{\text {tot }}(\omega) T\right], Y_{\text {th }}^{\text {tot }}(\omega)=G_{\text {th }}^{\text {tot }}+i \omega C_{h}$, and $Y_{\text {tot }}(\omega)=$ $G+i \omega C+1 /(i \omega L)$. The current through the inductor is $I_{L}(\omega)=V_{\mathrm{th}}(\omega) /(i \omega L)=\lambda_{I}(\omega) P_{\gamma}(\omega)$, where $\lambda_{I}(\omega)=\lambda_{V}(\omega) /$ $(i \omega L)$. Finally, we obtain the expressions of $V_{\mathrm{th}}(t)$ and $I_{L}(t)$,

$$
\begin{gathered}
V_{\mathrm{th}}(t)=\frac{1}{2 \pi} \int_{-\infty}^{\infty} d \omega \lambda_{V}(\omega) P_{\gamma}(\omega) e^{i \omega t}, \\
I_{L}(t)=\frac{1}{2 \pi} \int_{-\infty}^{\infty} d \omega \lambda_{I}(\omega) P_{\gamma}(\omega) e^{i \omega t} .
\end{gathered}
$$

The integrals in Eqs. (3) and (4) can easily be solved through the Cauchy residue theorem, but the general expressions are too long to be presented here. Rather, below we present some limiting cases.

Next, for the analyses of the various fluctuation processes present in the system, relevant for predicting the energy resolution of detection, we consider a Langevin noise circuit model. We denote $\delta T, \delta V$, and $\delta I_{L}$ as the temperature fluctuation on the absorber, voltage noise across the capacitor, and the current noise across the inductor. These noises are governed by the charge current noise $\delta I$ and heat current noise $\delta \dot{Q}_{J}$ across the thermoelectric junction and the heat current noise $\delta \dot{Q}_{\mathrm{q}-\mathrm{ph}}$ due to quasiparticle-phonon scattering. These noise terms satisfy the heat balance equation and the Kirchoff law for the noise terms in $\omega$ space $^{27}$ as

$$
\begin{gathered}
Y_{\mathrm{th}}^{\mathrm{tot}}(\omega) \delta T(\omega)=\delta \dot{Q}_{J}(\omega)+\delta \dot{Q}_{\mathrm{q}-\mathrm{ph}}(\omega)-\alpha \delta V(\omega), \\
Y_{\mathrm{tot}}(\omega) \delta V(\omega)=\delta I(\omega)-\frac{\alpha}{T} \delta T(\omega) .
\end{gathered}
$$

Solving Eqs. (5) and (6), we can obtain the voltage noise $\delta V(\omega)$ through the capacitor and the current noise through the inductor as $\delta I_{L}(\omega)=\delta V(\omega) /(i \omega L)$. We obtain the expressions of the noise correlations $\left\langle\delta V(t) \delta V\left(t^{\prime}\right)\right\rangle$ and $\left\langle\delta I_{L}(t) \delta I_{L}\left(t^{\prime}\right)\right\rangle$ in time domain through $4 \pi^{2}\left\langle\delta V(t) \delta V\left(t^{\prime}\right)\right\rangle=$ $\int_{-\infty}^{\infty} \int_{-\infty}^{\infty} d \omega d \omega^{\prime}\left\langle\delta V(\omega) \delta V\left(\omega^{\prime}\right)\right\rangle \quad e^{i \omega t} e^{i \omega^{\prime} t^{\prime}}$ and $4 \pi^{2}\left\langle\delta I_{L}(t)\right.$ $\left.\delta I_{L}\left(t^{\prime}\right)\right\rangle=\int_{-\infty}^{\infty} \int_{-\infty}^{\infty} d \omega d \omega^{\prime}\left\langle\delta I_{L}(\omega) \delta I_{L}\left(\omega^{\prime}\right)\right\rangle e^{i \omega t} e^{i \omega^{\prime} t^{\prime}}$. To obtain the second order correlations of these noise terms, we consider the intrinsic correlations of the detector as

$$
\begin{aligned}
& \left\langle\delta I(\omega) \delta I\left(\omega^{\prime}\right)\right\rangle=4 \pi k_{B} T G \delta\left(\omega+\omega^{\prime}\right), \\
& \left\langle\delta \dot{Q}_{J}(\omega) \delta \dot{Q}_{J}\left(\omega^{\prime}\right)\right\rangle=4 \pi k_{B} T^{2} G_{\mathrm{th}} \delta\left(\omega+\omega^{\prime}\right), \\
& \left\langle\delta I(\omega) \delta \dot{Q}_{J}\left(\omega^{\prime}\right)\right\rangle=-4 \pi k_{B} T \alpha \delta\left(\omega+\omega^{\prime}\right), \\
& \left\langle\delta \dot{Q}_{\mathrm{q}-\mathrm{ph}}(\omega) \delta \dot{Q}_{\mathrm{q}-\mathrm{ph}}\left(\omega^{\prime}\right)\right\rangle=4 \pi k_{B} T^{2} G_{\mathrm{q}-\mathrm{ph}} \delta\left(\omega+\omega^{\prime}\right), \\
& \left\langle\delta \dot{Q}_{J}(\omega) \delta \dot{Q}_{\mathrm{q}-\mathrm{ph}}\left(\omega^{\prime}\right)\right\rangle=0, \\
& \left\langle\delta I(\omega) \delta \dot{Q}_{\mathrm{q}-\mathrm{ph}}\left(\omega^{\prime}\right\rangle=0 .\right.
\end{aligned}
$$

Vanishing intrinsic correlations signify that the noises of the corresponding processes are independent. Finally, we obtain the following simplified expressions for the second order noise correlations as

$$
\begin{aligned}
\left\langle\delta V(t) \delta V\left(t^{\prime}\right)\right\rangle= & \frac{1}{2 \pi}\left(\frac{4 k_{B} T^{2} G_{\mathrm{th}}^{\mathrm{tot}}}{Z T}\right) \int_{-\infty}^{\infty} d \omega\left|\lambda_{V}(\omega)\right|^{2} \\
& \times\left[1+(1+Z T) \tau_{t h}^{2} \omega^{2}\right] e^{i \omega\left(t-t^{\prime}\right)}, \\
\left\langle\delta I_{L}(t) \delta I_{L}\left(t^{\prime}\right)\right\rangle= & \frac{1}{2 \pi}\left(\frac{4 k_{B} T^{2} G_{\mathrm{th}}^{\mathrm{tot}}}{Z T}\right) \int_{-\infty}^{\infty} d \omega\left|\lambda_{I}(\omega)\right|^{2} \\
& \times\left[1+(1+Z T) \tau_{t h}^{2} \omega^{2}\right] e^{i \omega\left(t-t^{\prime}\right)} .
\end{aligned}
$$

In Eqs. (7) and (8), $Z T=\alpha^{2} /\left(G_{\text {th }}^{\text {tot }} G T-\alpha^{2}\right)$ is the thermoelectric figure of merit and $\tau_{\text {th }}=C_{h} / G_{\text {th }}^{\text {tot }}$ is the thermal relaxation time. Our theoretical formalism for the current through the inductor and the second order noise correlation help us to analyze the optimum energy resolution of the thermoelectric detector in the calorimetric regime. As a result, we can find the condition for single photon detection in the far-infrared regime as shown below.

\section{RESULTS}

In this section, we discuss the results obtained from the formalism in Sec. II. First, we evaluate $I_{L}(t)$. Next, we obtain the optimal energy resolution in the calorimetric regime, with the idea of optimal filtering. This approach helps us analyzing a scheme for multiplexing the read-out. Finally, we predict the energy resolution in a SF based TED.

\section{A. Current through the inductor in the calorimetric regime}

Here, we analyze the behavior of the current through the inductor with respect to time at various circumstances in the calorimetric regime, that is, when $P_{\gamma}(t)=E \delta(t)$. Instead of the current, one could measure the voltage across the capacitor. The results are qualitatively similar, and the intrinsic energy resolution is the same in both cases.

In what follows, we denote the charge relaxation time $\tau_{R C}=C / G$ and LC time $\tau_{L C}=\sqrt{L C}$. For simplicity, we also define the corresponding frequencies by $\omega_{t h}=1 / \tau_{t h}$, $\omega_{R C}=1 / \tau_{R C}$, and $\omega_{L C}=1 / \tau_{L C}$. Using Eq. (4), first, we obtain $I_{L}(t)$ for finite $t \gg \tau_{R C}$, when the charge relaxation process is fastest, that is, for $\tau_{R C} \ll \tau_{t h}, \tau_{L C}$, as

$$
\begin{gathered}
I_{L}(t)=\frac{E \alpha}{C_{h} T(1+Z T)} \omega_{L C}^{2} \omega_{R C} \omega_{t h} \phi(t), \\
\phi(t)=\exp \left[\frac{-\omega_{t h} t}{1+Z T}\right]-\exp \left[\frac{-\omega_{L C}^{2}(1+Z T) t}{\omega_{R C}}\right] .
\end{gathered}
$$

Thus, in the case of fast charge relaxation, $I_{L}(t)$ tends to zero for $t \gtrsim \omega_{R L} /\left(\omega_{R C} \sqrt{1+Z T}\right)^{2}=L G /(1+Z T)$, but the initial decay is governed by the time scale $(Z T+1) \tau_{t h}$. Next, we obtain the expression of $I_{L}(t)$ for finite non-zero $t \gg \tau_{t h}$, 
when the thermal relaxation time is the fastest one, that is, $\tau_{\text {th }} \ll \tau_{R C}, \tau_{L C}$. It is

$$
\begin{gathered}
I_{L}(t)=\frac{4 E \alpha \omega_{t h} Y}{1+Z T} \exp \left[-\frac{\omega_{R C} t}{2(1+Z T)}\right] \sin \left(\frac{Y t}{1+Z T}\right), \\
2 Y=\sqrt{4 \omega_{L C}^{2}(1+Z T)^{2}-\omega_{R C}^{2}} .
\end{gathered}
$$

In this case, $I_{L}(t)$ is a decaying oscillatory function with a decaying time scale $2(Z T+1) \tau_{R C}$ if $Y$ is real. On the other hand, $I_{L}(t)$ simply decays, if $Y$ is imaginary. Finally, we analyze $I_{L}(t)$ for $t \gg \tau_{L C}$ for a high resonator frequency $\omega_{L C}$, that is, when $\tau_{L C} \ll \tau_{t h}, \tau_{R C}$. In this case,

$$
I_{L}(t)=\left(\frac{E \alpha \omega_{L C}^{2}}{C C_{h} L T}\right)\left[4 e^{-\omega_{R C} t / 2} \cos \left(\omega_{L C} t\right)-e^{-\omega_{t h} t}\right]
$$

Here $I_{L}(t)$ oscillates with the frequency $\omega_{L C}$. The oscillations decay within the charge relaxation time. These oscillations are visible especially when the first term dominates, i.e., $\tau_{R C} \gg \tau_{t h}$. Example time-dependent currents corresponding to these three regimes are shown in Fig. 2.

\section{B. Energy resolution in frequency domain with optimal filtering}

In what follows, we first optimize the energy resolution using the optimal filtering technique. ${ }^{28}$ From Eqs. (3) and (4), we obtain expressions for the thermoelectric voltage and current through the inductor as

$$
V_{t h}(t)=\frac{1}{2 \pi} \int_{-\infty}^{\infty} d \omega E \lambda_{V}(\omega) e^{i \omega t} p(\omega)
$$

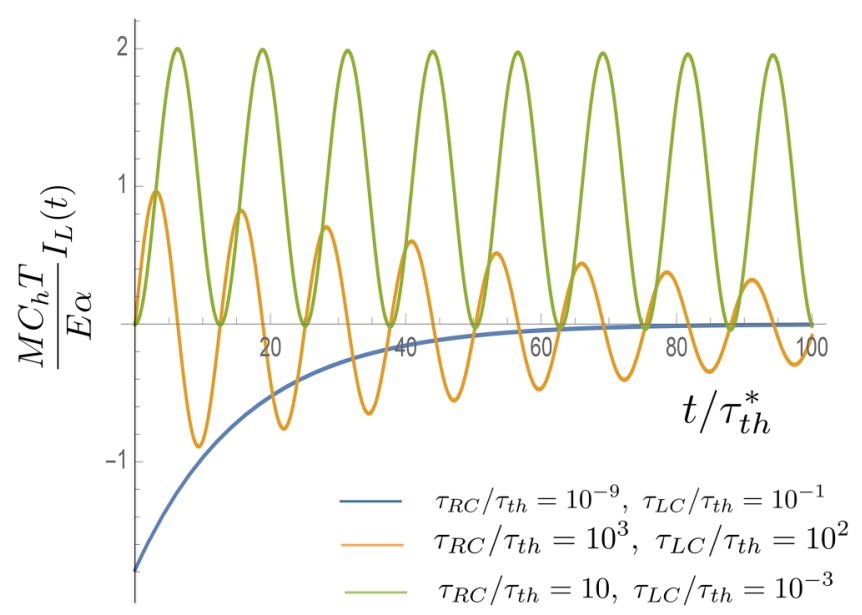

FIG. 2. Current $I_{L}(t)$ through the inductor in three parameter regimes: fast charge relaxation $\tau_{R C} \ll \tau_{t h}, \tau_{L C}$ (blue), fast thermal relaxation $\tau_{t h} \ll$ $\tau_{R C}, \tau_{L C}$ (orange), and high resonator frequency $\tau_{L C} \ll \tau_{t h}, \tau_{R C}$ (green). In all curves, $Z T=1$. $M$ is a scaling factor which takes different values for blue, orange, and green curves as $10^{8}, 10^{2}$, and 1 , respectively. The time scale $\tau_{t h}^{*}$ has the values $200 \tau_{t h}, 50 \tau_{t h}$, and $5 \times 10^{-4} \tau_{t h}$ for blue, orange, and green curves, respectively.

$$
I_{L}(t)=\frac{1}{2 \pi} \int_{-\infty}^{\infty} d \omega E \lambda_{I}(\omega) e^{i \omega t} p(\omega)
$$

where we have considered $P_{\gamma}(\omega)=E p(\omega)$ in Eqs. (3) and (4). Next, following Eqs. (7) and (8), we have the noise correlations for the thermoelectric voltage and current through the inductor as

$$
\begin{aligned}
& \left\langle\delta V(t) \delta V\left(t^{\prime}\right)\right\rangle=\frac{1}{2 \pi} \int_{-\infty}^{\infty} d \omega e_{V}^{2}(\omega) e^{i \omega\left(t-t^{\prime}\right)}, \\
& \left\langle\delta I_{L}(t) \delta I_{L}\left(t^{\prime}\right)\right\rangle=\frac{1}{2 \pi} \int_{-\infty}^{\infty} d \omega e_{I}^{2}(\omega) e^{i \omega\left(t-t^{\prime}\right)},
\end{aligned}
$$

where

$$
\begin{gathered}
e_{V}^{2}(\omega)=\left(\frac{4 k_{B} T^{2} G_{\mathrm{th}}^{\mathrm{tot}}}{Z T}\right)\left|\lambda_{V}(\omega)\right|^{2}\left[1+(1+Z T) \omega^{2} \tau_{t h}^{2}\right], \\
e_{I}^{2}(\omega)=\left(\frac{4 k_{B} T^{2} G_{\mathrm{th}}^{\mathrm{tot}}}{Z T}\right)\left|\lambda_{I}(\omega)\right|^{2}\left[1+(1+Z T) \omega^{2} \tau_{t h}^{2}\right] .
\end{gathered}
$$

From Fig. 2, we can see that when $\tau_{R C}$ is not the shortest time scale, $I_{L}(t)$ decays with oscillation as $t$ increases. Here our aim is to find the best estimate of $E$ in the presence of signal noise terms as Eqs. (15) and (16). Equations (15) and (16) indicate that the noise terms are correlated at different times and uncorrelated in frequency space, therefore, it is easier to make an analysis in frequency domain. Now, let us choose a weight function $W(\omega)$ in the frequency domain and therefore define the expected values for the signal and the corresponding noise terms as $\left\langle V_{t h}\right\rangle=\frac{1}{2 \pi} \int_{-\infty}^{\infty} d \omega W(\omega) E \lambda_{V}(\omega) p(\omega),\left\langle I_{L}\right\rangle=$ $\frac{1}{2 \pi} \int_{-\infty}^{\infty} d \omega W(\omega) E \lambda_{I}(\omega) p(\omega),\left\langle\delta V^{2}\right\rangle=\frac{1}{2 \pi} \int_{-\infty}^{\infty} d \omega|W(\omega)|^{2} e_{V}^{2}(\omega)$, and $\left\langle\delta I_{L}^{2}\right\rangle=\frac{1}{2 \pi} \int_{-\infty}^{\infty} d \omega|W(\omega)|^{2} e_{I}^{2}(\omega)$. Next, we can define the energy resolution of a generalized thermoelectric detector in terms of noise fluctuations and expected signals as $\Delta E=E \sqrt{\left\langle\delta V^{2}\right\rangle} /\left\langle V_{t h}\right\rangle=E \sqrt{\left\langle\delta I_{L}^{2}\right\rangle} /\left\langle I_{L}\right\rangle^{28}$ At this point, as we desire to have the maximum value of signal to noise ratio, and hence the minimum energy resolution, we need to search for an optimal filter, that is, an optimal $W(\omega)$. The desired optimal filter can be found out by finding a zero of the functional derivative of $\Delta E$ with respect to $W(\omega) .^{28}$ The optimal filter is $W(\omega)=E \lambda_{V}(-\omega) p(\omega) / e_{V}^{2}(\omega)=$ $E \lambda_{I}(-\omega) p(\omega) / e_{I}^{2}(\omega)$. Using this weight function, we obtain an expression of the optimum energy resolution through such an optimal filter in the calorimetric regime, that is, when $P_{\gamma}(t)=E \delta(t)$ or equivalently $p(\omega)=1$, given by

$$
\Delta E_{\mathrm{opt}}^{(\mathrm{Fil})}=N E P \sqrt{\tau_{\mathrm{eff}}}
$$

where $N E P^{2}=4 k_{B} T^{2} G_{t h}^{t o t} / Z T$ and $\tau_{\text {eff }}=\tau_{t h} \sqrt{1+Z T}$. In Eq. (19), the effective time constant, $\tau_{\text {eff }}$ is affected by $Z T$. This is the generalization of the energy resolution obtained earlier for a thermoelectric detector in the calorimetric regime with a non-zero $Z T^{16}$

In the above analysis about energy resolution with the idea of optimal filtering, we have not considered the added 
noise in amplification, described by the low-frequency power spectral density $S_{A}$. Now, in the measured signal, we consider an effect due to noise term $\delta I_{A}(t)$ of a current amplifier, where amplifier noise term is uncorrelated with other intrinsic noise terms of the detector. We assume the amplifier current noise fluctuation in the calorimetric regime as $\left\langle\delta I_{A}(t) \delta I_{A}\left(t^{\prime}\right)\right\rangle=\frac{1}{2 \pi} \int_{-\infty}^{\infty} d \omega S_{A} e^{i \omega\left(t-t^{\prime}\right)}$. Therefore, in order to obtain the optimal energy resolution, we design the optimal filter by including the effect due to amplifier current noise term. As a result, we get the optimal energy resolution $\Delta E_{\mathrm{opt}}^{(\mathrm{Fil})}=N E P_{\text {tot }} \sqrt{\tau_{\mathrm{eff}}^{\mathrm{tot}}}$, where $N E P_{\text {tot }}^{2}=4 k_{B} T^{2} G_{\text {th }}^{\text {tot }} / Z T_{\text {tot }}$ and $\tau_{\text {eff }}^{\text {tot }}=\tau_{t h} \sqrt{1+Z T_{\text {tot }}}$. Here $Z T_{\text {tot }}^{-1}=Z T^{-1}+Z T_{A}^{-1}$, where $1 / Z T_{A}=S_{A} G_{t h}^{\text {tot }} /\left(2 k_{B} \alpha^{2}\right)$. The effect of the amplifier can hence be disregarded if $Z T / Z T_{A}=S_{A}(1+Z T) /\left(2 G k_{B} T\right) \ll 1$.

\section{Comment about multiplexing}

In this section, we consider a practical multiplexing case by doing a numerical experiment. For this, we define the filtered signal in the calorimetric regime from Sec. III B as

$$
I_{L}^{(\mathrm{Fil})}(t)=\frac{E}{2 \pi} \int_{-\infty}^{\infty} d \omega W(\omega) \lambda_{I}(\omega) e^{i \omega t}
$$

Now, in Eq. (20), if we consider $W(\omega)$ to be the optimal filter as obtained in Sec. III B, then we have

$$
\begin{aligned}
& I_{L}^{(\mathrm{Fil})}(t)=A \exp \left[-t /\left(\tau_{t h} \sqrt{1+Z T_{\text {tot }}}\right)\right] \\
& A=\frac{E^{2} Z T_{\text {tot }}}{8 k_{B} T^{2} G_{\text {th }}^{\text {tot }}}\left(1+Z T_{\text {tot }}\right)^{-1 / 2} .
\end{aligned}
$$

Therefore, for the optimal filter, the plot of $-\ln I_{L}^{\text {(Fil) }}(t)$ vs $t$ is simply a straight line with the slope $\tau_{t h} \sqrt{1+Z T_{\text {tot }}}$. On the other hand, for any random filter, $I_{L}^{(\mathrm{Fil})}(t)$ does not have simple decaying form but further oscillates in time. As a result, the time-averaged current becomes very small. From this feature of $I_{L}^{(\text {Fil })}(t)$, we can identify the pixel for which the designed filter is approximately optimal. In Fig. 3, we represent $I_{L}^{(\mathrm{Fil})}(t)$ when the filter is optimal (blue curve) and when the filter is not optimal (orange curve).

\section{Energy resolution of the SF based TED}

Above discussion is valid for a generic TED. In what follows, we evaluate the energy resolution $\Delta E_{\mathrm{opt}}^{(\mathrm{Fil})}$ of an SF based TED, disregarding the effect of amplifier noise in the measured signal. In the tunneling limit, where higher-order processes such as Andreev reflection can be disregarded, the coefficients of the thermoelectric detector $\operatorname{are}^{18,19}$

$$
G=G_{T} \int_{-\infty}^{\infty} d E \frac{N_{0}(E)}{4 k_{B} T \cosh ^{2}\left(\frac{E}{2 k_{B} T}\right)}
$$

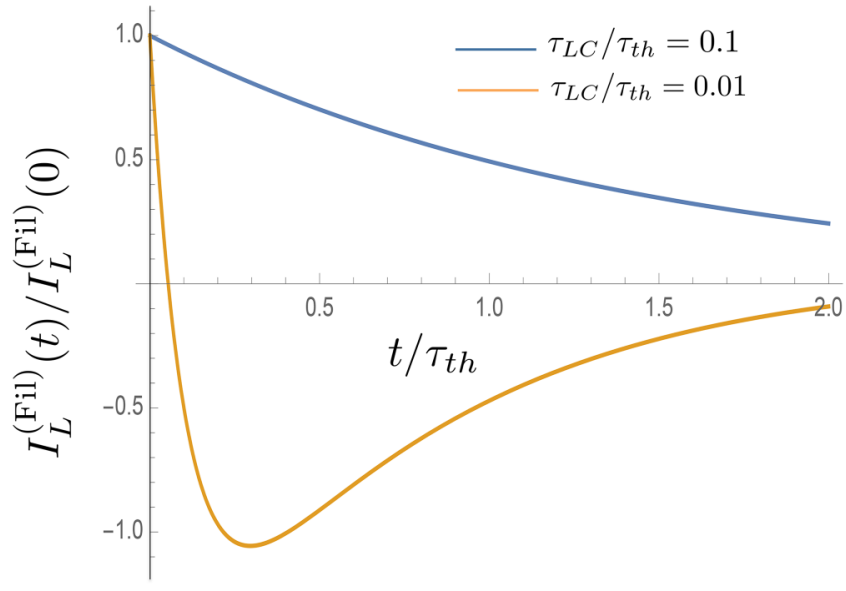

FIG. 3. Filtered current $I_{L}^{(\mathrm{Fil})}(t)$ through the inductor. In all curves, $\tau_{R C} / \tau_{t h}=$ $10^{-3}$ and $Z T_{\text {tot }}=1$. In the blue curve $W(\omega)$, we choose the optimal filter. On the other hand, for the orange curve, we consider the $W(\omega)$ is equal to the optimal filter of the blue curve.

$$
\begin{gathered}
G_{t h}=\frac{G_{T}}{e^{2}} \int_{-\infty}^{\infty} d E \frac{E^{2} N_{0}(E)}{4 k_{B} T^{2} \cosh ^{2}\left(\frac{E}{2 k_{B} T}\right)}, \\
\alpha=\frac{P G_{T}}{2 e} \int_{-\infty}^{\infty} d E \frac{E N_{z}(E)}{4 k_{B} T \cosh ^{2}\left(\frac{E}{2 k_{B} T}\right)} .
\end{gathered}
$$

Here $P=\left(G_{\uparrow}-G_{\downarrow}\right) /\left(G_{\uparrow}+G_{\downarrow}\right)$ is the spin polarization, $G_{\sigma}$ is the normal-state conductance for spin $\sigma$, $N_{0}(E)=\left(N_{\uparrow}+N_{\downarrow}\right) / 2$, and $\quad N_{z}(E)=N_{\uparrow}-N_{\downarrow} \quad$ are $\quad$ the spin-averaged and spin-difference density of states of the superconductor, normalized to the normal-state density of states, $v_{F}$, at the Fermi level. Here $N_{\uparrow / \downarrow}=N_{S}(E \pm h)$ with $N_{S}(E)=\operatorname{Re}\left[|\mathrm{E}+\mathrm{i} \Gamma| / \sqrt{(\mathrm{E}+\mathrm{i} \Gamma)^{2}-\Delta^{2}}\right], \quad h \quad$ is the spin splitting exchange field and $\Gamma \ll \Delta$ describes pairbreaking inside the superconductor. The heat capacity of the absorber with the volume $\Omega$ of the superconductor is $C_{h}=v_{F} \Omega e^{2} G_{t h} / G_{T}{ }^{18}$ Finally, the electron-phonon heat conductance is obtained from ${ }^{18,21}$

$$
\begin{aligned}
G_{q-p h}= & \frac{\Sigma \Omega}{96 \zeta(5) k_{B}^{6} T^{2}} \int_{-\infty}^{\infty} d E E \int_{-\infty}^{\infty} d \omega \omega^{2}|\omega| \\
& \times L_{E, E+\omega} F_{E, \omega}, \\
L_{E, E^{\prime}}= & \frac{1}{2} \sum_{\sigma=\uparrow, \downarrow} N_{\sigma}(E) N_{\sigma}\left(E^{\prime}\right) \\
& \times\left[1-\Delta^{2} /\left[(E+\sigma h)\left(E^{\prime}+\sigma h\right)\right]\right] \\
F_{E, \omega}= & -\frac{1}{2}\left[\sinh \left(\frac{\omega}{2 k_{B} T}\right) \cosh \left(\frac{E}{2 k_{B} T}\right)\right. \\
& \left.\quad \times \cosh \left(\frac{E+\omega}{2 k_{B} T}\right)\right]^{-1} .
\end{aligned}
$$


In Eq. (24a), $\Sigma$ is the material dependent electron-phonon coupling constant and $\zeta(5)$ is the Riemann zeta function. For $k_{B} T \ll \Delta-h$, the thermoelectric coefficients have the analytical estimates ${ }^{18,19}$

$$
\begin{gathered}
G \approx G_{T} \sqrt{2 \pi \tilde{\Delta}} \cosh (\tilde{h}) e^{-\tilde{\Delta}}, \\
G_{t h} \approx \\
\frac{k_{B} G_{T} \Delta}{e^{2}} \sqrt{\frac{\pi}{2 \tilde{\Delta}}} e^{-\tilde{\Delta}}\left[e^{\tilde{h}}(\tilde{\Delta}-\tilde{h})^{2}\right. \\
\left.+e^{-\tilde{h}}(\tilde{\Delta}+\tilde{h})^{2}\right], \\
\alpha \approx \frac{P G_{T}}{e} \sqrt{2 \pi \tilde{\Delta}} e^{-\tilde{\Delta}}[\Delta \sinh (\tilde{h})-h \cosh (\tilde{h})], \\
G_{q-p h} \approx \frac{\Sigma \Omega}{96 \zeta(5)} T^{4}\left[\cosh (\tilde{h}) e^{-\tilde{\Delta}} f_{1}(\tilde{\Delta})\right. \\
\left.+\pi \tilde{\Delta}^{5} e^{-2 \tilde{\Delta}} f_{2}(\tilde{\Delta})\right],
\end{gathered}
$$

where $\tilde{h}=h / k_{B} T$ and $\tilde{\Delta}=\Delta / k_{B} T$. In Eq. (28), the terms $f_{1}$ and $f_{2}$ represent the scattering and recombination processes. The functions $f_{1}(x)=\sum_{n=0}^{3} C_{n} / x^{n}$ and $f_{2}(x)=\sum_{n=0}^{2} B_{n} / x^{n}$, where $C_{0}=440, C_{1}=-500, C_{2}=1400, C_{3}=-4700$, $B_{0}=64, B_{1}=144$, and $B_{2}=258$. The analytical estimate of the $\Delta E_{\mathrm{opt}}^{(\mathrm{Fil})}$ can now be obtained by substituting Eqs. (25)-(28) in Eq. (19). As at low temperatures, the scattering contribution dominates over recombination in the quasiparticle-phonon heat conductance, we neglect the recombination process and assume $f_{1}(x) \approx 400$ for $k_{B} T \lesssim$ $0.1 \Delta$ to obtain a simplified analytical estimate of the energy resolution $\Delta E_{\mathrm{opt}}^{(\mathrm{Fil})}$ of the SF based TED.

$$
\begin{aligned}
& \Delta E_{\mathrm{opt}}^{(\mathrm{Fil})} \approx\left[\sqrt{4 v_{\mathrm{F}} \Omega k_{B}^{3} T^{3} \chi / Z T}\right](1+Z T)^{1 / 4}, \\
& \chi=(2 \pi \tilde{\Delta})^{1 / 2} e^{-\tilde{\Delta}}\left[\left(\tilde{\Delta}^{2}+\tilde{h}^{2}\right) \cosh (\tilde{h})\right. \\
& -2 \tilde{\Delta} \tilde{h} \sinh (\tilde{h})] \text {, } \\
& Z T \approx \frac{P^{2}}{1-P^{2}+\frac{\tilde{\Delta}^{2}+Z_{\mathrm{spur}} \cosh ^{2}(\tilde{h})}{[\tilde{h} \cosh (\tilde{h})-\tilde{\Delta} \sinh (\tilde{h})]^{2}},} \\
& Z_{\mathrm{spur}}=\frac{e^{2} \Sigma \Omega \Delta^{3}}{G_{T} k_{B}^{5}} \tilde{\Delta}^{-4} \frac{220}{96 \zeta(5)} .
\end{aligned}
$$

In the following, we use the formulas of Eqs. (19)-(24c) to find $\Delta E_{\mathrm{opt}}^{(\mathrm{Fi})}$ as a function of the exchange field and the bath temperature of the SF based TED. We also compare the numerical results with the analytical estimate in Eq. (29). We consider an $\mathrm{Al}$ absorber of volume $\Omega=10^{-19} \mathrm{~m}^{3}$ and the superconducting critical temperature at zero exchange field $T_{c}=1.2 \mathrm{~K},,^{29} v_{\mathrm{F}}=10^{47} \mathrm{~J}^{-1} \mathrm{~m}^{-3}$, and $G_{T}=5 \times 10^{-4}$ $e^{2} \Sigma \Omega \Delta^{3} / k_{B}^{5} \sim 25 \mu \mathrm{S}$. ${ }^{18}$ With these choices, we get an overall

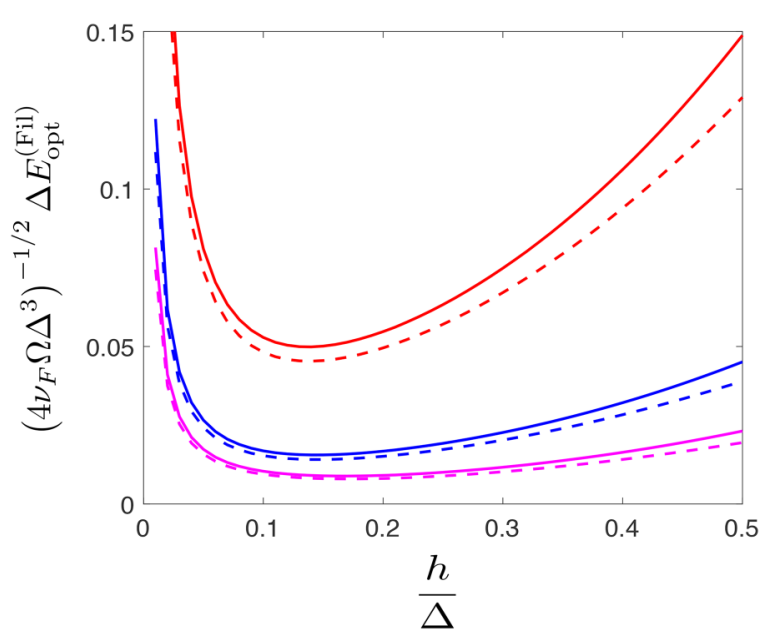

FIG. 4. Energy resolution after optimal filtering as a function of exchange field for $\Gamma=10^{-4} \Delta, k_{B} T=0.1 \Delta$, and $G_{T}=5 \times 10^{-4} e^{2} \Sigma \Omega \Delta^{3} / k_{B}^{5}$, where red, blue, and magenta lines, respectively, represent the plots for $P=0.2$, $P=0.6$, and $P=0.9$. The solid lines are obtained numerically, whereas the dashed lines are the analytical estimates from Eq. (29) for the corresponding situations.

scaling factor $\sqrt{4 v_{F} \Omega \Delta^{3}}=20 \mathrm{meV}$. This factor is used in Figs. 4 and 5 as the unit energy resolution. We thus find that the optimal energy resolution at $k_{B} T=0.1 \Delta$, corresponding to $T=200 \mathrm{mK}$ for Al, can be below $1 \mathrm{meV}$. This corresponds to single-photon resolution at frequencies $f=\Delta E_{\mathrm{opt}}^{\mathrm{Fil}} /(2 \pi \hbar)$ above $240 \mathrm{GHz}$. Figure 5 shows the corresponding temperature dependence of the optimal energy resolution (solid lines). The analytical estimate in Eq. (29) fits the numerics up to $k_{B} T \lesssim 0.1 \Delta$. Above this, the quasiparticle-phonon recombination process starts affecting the results. Note that for this range of energy resolution $Z T$ need not be extremely high. For example, the largest $Z T$ in the cases $P=0.6$ and $P=0.9$ in Fig. 4 are 0.52 and 3.45, respectively.

Let us again discuss the added noise in current amplification. A good cryogenic SQUID amplifier can reach

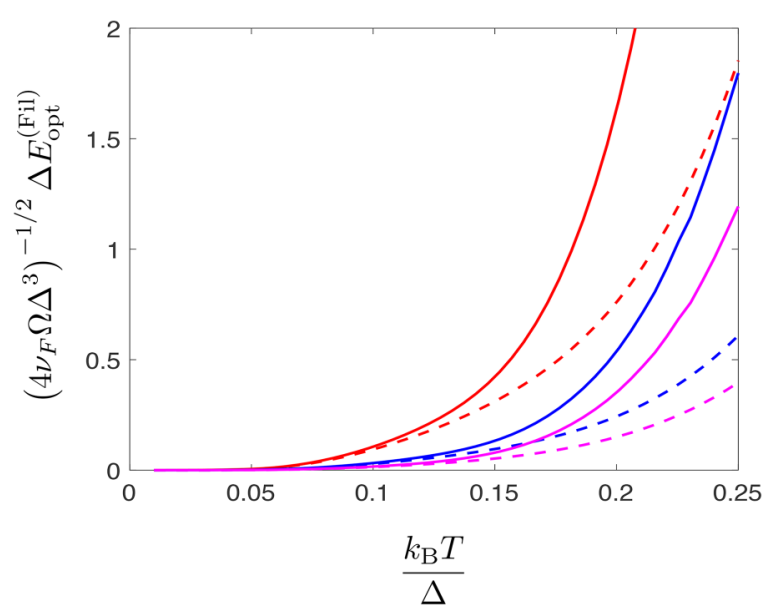

FIG. 5. Energy resolution after optimal filtering as a function of temperature for $\Gamma=10^{-4} \Delta, h=0.4 \Delta$, and $G_{T}=5 \times 10^{-4} e^{2} \Sigma \Omega \Delta^{3} / k_{B}^{5}$, where red, blue, and magenta lines, respectively, represent the plots for $P=0.2, P=0.6$, and $P=0.9$. The solid lines are obtained numerically, whereas the dashed lines are the analytical estimates from Eq. (29) for the corresponding situations. 
$S_{A} \sim 0.3(\mathrm{fA})^{2} / \mathrm{Hz} .{ }^{30}$ With the above-chosen tunnel conductance and superconducting gap $\Delta$, this would translate into $Z T / Z T_{A} \approx 2 \times 10^{-4} \times(1+Z T) G_{T} \Delta /\left(G k_{B} T\right)$. This becomes of the order of unity or larger for $k_{B} T \lesssim 0.1 \Delta$. Below that temperature, it would be advantageous to either measure the voltage instead of the current or use higher-conductance junctions. Contrary to the noise equivalent power in bolometers, ${ }^{18}$ this does not deteriorate the energy resolution. However, increasing the contact transparency may be challenging especially with $\mathrm{Al} / \mathrm{EuS}$ based spin-filter junctions.

Outside the linear response regime, where $\Delta T$ becomes large, another noise mechanism related to shot noise ${ }^{31}$ starts to play a role. This mechanism becomes relevant in the nonlinear regime of the detector, and its analysis is left for further work.

\section{CONCLUSIONS}

In this work, we present the first full noise analysis of a generic thermoelectric detector TED, especially including the possibility of a high thermoelectric figure of merit. In particular, we show that TEDs based on superconductorferromagnet systems may rival the best transition edge sensor (TES)-type calorimeters, reaching wide-band energy resolution below $1 \mathrm{meV}$ (with unit quantum efficiency). Hence, such SF TEDs present a viable alternative for TES devices, especially in the case of large arrays where the lack of required probe power leads to reduced heating and simplified design of the detectors.

\section{ACKNOWLEDGMENTS}

We thank Ilari Maasilta for discussions. This project was supported by the Academy of Finland Key Funding project (Project No. 305256) and the Center of Excellence program (Project No. 284594).

${ }^{1}$ K. Irwin and G. Hilton, "Transition-edge sensors," in Cryogenic Particle Detection, edited by C. Enss (Springer, Berlin, 2005), pp. 63-150.

${ }^{2}$ A. Luukanen, R. Appleby, M. Kemp, and N. Salmon, in Terahertz Spectroscopy and Imaging, edited by K. E. Peiponen, A. Zeitler, and M. Kuwata-Gonokami (Springer, Berlin, 2013), pp. 491-520.

${ }^{3}$ J. N. Ullom and D. A. Bennett, Supercond. Sci. Technol. 28, 084003 (2015).

${ }^{4}$ M. R. J. Palosaari, M. Käyhkö, K. M. Kinnunen, M. Laitinen, J. Julin, J. Malm, T. Sajavaara, W. B. Doriese, J. Fowler, C. Reintsema, D. Swetz, D. Schmidt, J. N. Ullom, and I. J. Maasilta, Phys. Rev. Appl. 6, 024002 (2016).
${ }^{5}$ D. Hanson et al. (SPTpol Collaboration), Phys. Rev. Lett. 111, 141301 (2013).

${ }^{6}$ M. Madhavacheril et al. (Atacama Cosmology Telescope Collaboration), Phys. Rev. Lett. 114, 151302 (2015).

${ }^{7}$ F. Giazotto, T. T. Heikkilä, A. Luukanen, A. M. Savin, and J. P. Pekola, Rev. Mod. Phys. 78, 217 (2006).

${ }^{8}$ E. N. Grossman, D. G. McDonald, and J. E. Sauvageau, IEEE Trans. Magn. 27, 2677 (1991).

${ }^{9}$ N. Bluzer and M. G. Forrester, Opt. Eng. 33, 33 (1994).

${ }^{10}$ A. V. Sergeev, V. V. Mitin, and B. S. Karasik, Appl. Phys. Lett. 80, 817 (2002).

${ }^{11}$ F. Giazotto, T. T. Heikkilä, G. P. Pepe, P. Helistö, A. Luukanen, J. P. Pekola, Appl. Phys. Lett. 92, 162507 (2008).

${ }^{12}$ J. Govenius, R. E. Lake, K. Y. Tan, and M. Möttönen, Phys. Rev. Lett. 117, 030802 (2016).

${ }^{13}$ D. Farrah, K. E. Smith, D. Ardila, C. M. Bradford, M. Dipirro, C. Ferkinhoff, J. Glenn, P. Goldsmith, D. Leisawitz, T. Nikola et al., e-print arXiv:1709.02389 (2017).

${ }^{14}$ R. C. Jones, J. Opt. Soc. Am. 37, 879 (1947).

${ }^{15}$ A. Varpula, A. V. Timofeev, A. Shchepetov, K. Grigoras, J. Hassel, J. Ahopelto, M. Ylilammi, and M. Prunnila, Appl. Phys. Lett. 110, 262101 (2017).

${ }^{16}$ D. V. Vechten, K. Wood, G. Fritz, A. Gyulamiryan, V. Nikogosyan, N. Giordano, T. Jacobs, and A. Gulian, in Eighteenth International Conference on Thermoelectrics (IEEE, 1999), pp. 477-480.

${ }^{17}$ G. Skoblin, J. Sun, and A. Yurgens, Appl. Phys. Lett. 112, 063501 (2018).

${ }^{18}$ T. T. Heikkilä, R. Ojajärvi, I. J. Maasilta, F. Giazotto, and F. S. Bergeret, e-print arXiv:1709.08856 [cond-mat.supr-con] (2017).

${ }^{19}$ A. Ozaeta, P. Virtanen, F. S. Bergeret, and T. T. Heikkilä, Phys. Rev. Lett. 112, 057001 (2014).

${ }^{20}$ S. Kolenda, M. J. Wolf, and D. Beckmann, Phys. Rev. Lett. 116, 097001 (2016).

${ }^{21}$ F. S. Bergeret, M. Silaev, P. Virtanen, and T. T. Heikkilä, e-print arXiv:1706.08245 [cond-mat.supr-con] (2017).

${ }^{22}$ A. M. Clogston, Phys. Rev. Lett. 9, 266 (1962).

${ }^{23}$ J. A. X. Alexander, T. P. Orlando, D. Rainer, and P. M. Tedrow, Phys. Rev. B 31, 5811 (1985).

${ }^{24}$ E. Strambini, V. N. Golovach, G. De Simoni, J. S. Moodera, F. S. Bergeret, and F. Giazotto, Phys. Rev. Materials 1, 054402 (2017).

${ }^{25}$ J. S. Moodera, X. Hao, G. A. Gibson, and R. Meservey, Phys. Rev. Lett. 61, 637 (1988).

${ }^{26}$ Throughout this work, we use the Fourier transform convention as $F(t)=\frac{1}{2 \pi} \int_{-\infty}^{\infty} d \omega F(\omega) e^{i \omega t}$.

${ }^{27}$ Y. Blanter and M. Buttiker, Phys. Rep. 336, 1 (2000).

${ }^{28} \mathrm{D}$. McCammon, "Thermal equilibrium calorimeters-an introduction," in Cryogenic Particle Detection, edited by C. Enss (Springer, Berlin, 2005), pp. 1-34.

${ }^{29}$ B. T. Matthias, T. H. Geballe, and V. B. Compton, Rev. Mod. Phys. 35, 1 (1963).

${ }^{30}$ N. Beev and M. Kiviranta, Cryogenics 57, 129 (2013).

${ }^{31}$ T. T. Heikkilä, The Physics of Nanoelectronics: Transport and Fluctuation Phenomena at Low Temperatures (Oxford University Press, 2013), Vol. 21 